\title{
UPOINT System: A Diagnostic/Therapeutic Algorithm for Chronic Prostatitis/Chronic Pelvic Pain Syndrome
}

\author{
Phil Hyun Song \\ Department of Urology, College of Medicine, Yeungnam University, Daegu, Korea
}

\begin{abstract}
Chronic prostatitis/chronic pelvic pain syndrome (CP/CPPS) is a common condition that significantly affects the quality of life. On the other hand, urologists find it challenging to treat this disorder effectively. To date, the Urinary, Psychosocial, Organ-Specific, Infection, Neurological/Systemic, and Tenderness (UPOINT) system is the only classification tool that can improve the treatment outcomes significantly compared to monotherapy. This review focused on the most recent advances in the diagnosis and treatment of CP/CPPS.
\end{abstract}

Keywords: Chronic prostatitis with chronic pelvic pain symdrome; Phenotype; Therapeutics

Copyright (c) 2020, Korean Association of Urogenital Tract Infection and Inflammation. All rights reserved. (i) \$ This is an open access article distributed under the terms of the Creative Commons Attribution Non-Commercial License (http://creativecommons.org/licenses/by-nc/4.0) which permits unrestricted non-commercial use, distribution, and reproduction in any medium, provided the original work is properly cited.
Received: 21 July, 2020

Revised: 14 August, 2020

Accepted: 15 August, 2020

\section{INTRODUCTION}

Chronic prostatitis/chronic pelvic pain syndrome (CP/ CPPS) is a common urological condition that has a significant negative impact on the quality of life and poses a financial burden similar to other chronic illnesses, such as diabetes mellitus, Crohn's disease, and myocardial infarction [1]. The characterization and treatment of prostatitis have been challenging for urologists, and there has been no validated classification system for this condition for decades. In 1999 , the National Institutes of Health (NIH) developed a classification system based on the clinical syndromes to define this disease better (Table 1). This system can classify prostatitis into the following four categories: acute bacterial prostatitis, chronic bacterial prostatitis, CP/CPPS, and asymptomatic prostatitis [2].

Accounting for more than $90 \%$ of all urological outpatients, $\mathrm{CP} / \mathrm{CPPS}$ is among the most common urologic disorders in men younger than 50 years. Moreover, the condition is often poorly understood, and its management is challenging [3].
This may be correlated with the heterogeneity of CP/CPPS Owing to the clinical diversity of patients with this disease, each patient should be classified according to the unique clinical phenotype. The Urinary, Psychosocial, Organ-Specific, Infection, Neurological/Systemic, and Tenderness (UPOINT) system is a diagnostic/therapeutic algorithm developed in 2009 [4]. This review article presents the development of the UPOINT phenotyping system and the latest treatment methods for CP/CPPS based on this system.

\section{DEVELOPMENT OF THE UPOINT}

The UPOINT system is used to clinically classify a patient's symptoms into six broad categories to facilitate individualized and multimodal therapy. Each domain is treated differently, thereby allowing for a multimodal approach. This system was found to be effective in assessing the symptom severity in chronic pelvic pain. That is, patients with more positive domains have a longer duration of symptoms and a higher National Institutes of Health Chronic Prostatitis 
Table 1. National Institute of Health (NIH) chronic prostatitis/chronic pelvic pain syndrome classification system

\begin{tabular}{lll}
\hline \multicolumn{1}{c}{ Traditional classification } & \multicolumn{1}{c}{ NIH classification } & Description \\
\hline $\begin{array}{l}\text { Acute bacterial prostatitis } \\
\text { Chronic bacterial prostatitis } \\
\text { Nonbacterial prostatitis }\end{array}$ & Category I & Category II \\
Category IIIA (inflammatory CP/CPPS) & $\begin{array}{l}\text { Acute infection of the prostate } \\
\text { Chronic infection of the prostate } \\
\text { Significant number of leukocytes in expressed prostatic fluid or } \\
\text { semen } \\
\text { Insignificant number of leukocytes in expressed prostatic fluid } \\
\text { or semen }\end{array}$ \\
Category IIIB (noninflammatory CP/CPPS) & Category IV (asymptomatic inflammatory CP) & $\begin{array}{c}\text { Leukocytes and or bacteria in expressed prostatic fluid or } \\
\text { semen without the presence of pelvic pain/urinary symptoms }\end{array}$ \\
\hline
\end{tabular}

CP/CPPS: chronic prostatitis/chronic pelvic pain syndrome.

Symptom Index (NIH-CPSI) score [4]. Magri et al. [5] evaluated the UPOINT phenotyping system. The results showed that $77.5 \%$ of 914 patients experienced a significant decrease in the CPSI score at 18 months. Moreover, a Swedish study validated the correlation between the number of positive domains and the NIH-CPSI score [6]. Although further validation studies will be needed, the UPOINT clinical phenotyping system provides a useful and clinically relevant framework for multimodal therapy for the management of $\mathrm{CP} / \mathrm{CPPS}$.

\section{NOVEL TREATMENTS}

\section{Urinary}

The efficacy of several alpha-blockers used in monotherapy has been assessed, but the results varied. Nickel et al. [7] conducted a randomized controlled trial (RCT) and reported that tamsulosin $0.4 \mathrm{mg}$ daily significantly improved the NIH-CPSI scores across multiple domains compared to the placebo. In another study, silodosin $4 \mathrm{mg}$ reduced the NIH-CPSI scores compared to the placebo [8]. Alpha-blockers may be beneficial when used in multimodal therapy. Owing to the heterogeneity of previous studies, however, the authors of a recent study could not recommend alpha-blockers as first-line monotherapy [9].

Some studies reported that phosphodiesterase-5 inhibitors could improve the lower urinary tract symptoms (LUTS) by inhibiting nitric oxide synthase. Kong et al. [10] reported that the use of mirodenafil in combination with levofloxacin versus levofloxacin alone improved the NIH-CPSI score by -7.2 and $-3.2(\mathrm{p}<0.05)$, respectively.

\section{Psychosocial}

Cannabis has been used in other chronic pain syndromes for symptom relief. Tripp et al. [11] performed a study on men with CP/CPPS who visited clinics and shared their experience with cannabis online. In total, 342 participants responded to questions regarding the baseline NIH-CPSI scores, symptom improvement with cannabis usage, and side effects. The results showed that $57 \%$ of clinics and $63 \%$ of online participants believed that cannabis was effective to some extent in treating CP/CPPS symptoms. Moreover, it may play a role in the management of CP/CPPS. On the other hand, multiple studies revealed an association between regular cannabis use and low sperm concentration and total sperm count as well as decreased sperm function [12,13]. $\mathrm{CP} / \mathrm{CPPS}$ is a disease common in men younger than 50 years old. Thus, male fertility and the desire for future offspring should be considered before initiating therapy with cannabis [5].

\section{Organ-Specific}

Phytotherapies, such as quercetin, saw palmetto, and cernilton, have been used owing to their success in improving the symptoms of patients with CP/CPPS [14]. In another study on the effects of pollen extract, compared to patients $(n=10)$ in the placebo group, patients in the experimental group experienced an improvement in pain and LUTS based on a symptom questionnaire survey [15].

OM-89 is a novel oral immunostimulatory agent developed from lysed pathogenic Escherichia coli bacteria. Wagenlehner et al. [16] conducted a randomized, double-blind placebo-controlled study on 185 patients who received either OM-89 or a placebo. The results showed that the NIH-CPSI score decreased by $40.5 \%$ and $44.0 \%$ in the treatment and placebo groups, respectively. Moreover, the positive response rates were $67 \%$ and $64.3 \%$, respectively. On the other hand, the results did not differ significantly.

Increasing evidence suggests that low-intensity pulsed ultrasound (LIPUS) can inhibit inflammation and pain by 
regulating the cyclooxygenase pathway. Li et al. [17] performed a randomized study on $96 \mathrm{CP} / \mathrm{CPPS}$ patients who either received treatment with transperineal ultrasonography daily for two weeks or underwent a sham treatment. The results showed that the two groups differed significantly in terms of the total NIH-CPSI and pain and urinary symptom scores after the LIPUS treatment.

\section{Infection}

Antibiotics are often used for patients with CP/CPPS. On the other hand, the use of antibiotics has been controversial in the absence of infection. Zhou et al. [18] reported that the mean NIH-CPSI score decreased by 18.5 points in the tetracycline group. Nevertheless, two other RCTs compared the efficacy of ciprofloxacin or levofloxacin for six weeks versus placebo in the treatment of CP/CPPS. The results did not reveal any significant difference in the changes in $\mathrm{NIH-CPSI}$ scores [14]. Hence, there is no sufficient evidence supporting the efficacy of antibiotics in the primary treatment of CP/CPPS [1,9].

Anti-inflammatory medications have been a therapeutic option for CPPS. On the other hand, the outcomes have varied. A previous study showed that celecoxib improves the condition of patients. On the other hand, deterioration was observed two weeks after therapy [19]. In another RCT on pentosan polysulfate sodium, there was no significant improvement in the Clinical Global Improvement and total NIH-CPSI scores [15].

New second-generation drugs, including beclomethasone dipropionate, are aimed to decrease the systemic activity while maintaining local efficacy. Hence, they have attracted increasing interest. Bozzini et al. [20] performed a study on 180 men with CP/CPPS, who received beclomethasone dipropionate suppositories. Of these patients, 136 received a Serenoa repens extract. The results showed that the men in the steroid group and those in the steroid and $S$. repens-combined group experienced significant decreases in voiding frequency ( -3.55 vs. -3.68 voids per day, respectively) and increases in uroflowmetry (3.26 vs. 5.61 $\mathrm{ml} / \mathrm{s}$, respectively). Moreover, both groups experienced an improvement in perineal pain based on the visual analog scale. Therefore, beclomethasone dipropionate suppositories were considered safe and were associated with improvement in LUTS and pain.

Kotb et al. [21] examined the efficacy of antifungals and urinary alkalization with potassium citrate in 1,000 men who did not respond to four weeks of treatment with antibiotics and alpha-blockers 31. Of 1,000 men, 803 experienced at least $80 \%$ improvement in their urinary and pain symptoms. A low initial prostate-specific antigen level and age were associated with a better response to antifungals.

\section{Neurological/Systemic}

Neuromodulatory techniques have shown promising results in the treatment of $\mathrm{CP} / \mathrm{CPPS}$. In a randomized, placebo-controlled, double-blind trial, Kessler et al. [22] examined the effects of sono-electromagnetic (SEM) therapy in the treatment of refractory $\mathrm{CP} / \mathrm{CPPS}$. The patients had received other therapies, including antibiotics, nonsteroidal anti-inflammatory drugs, and alpha-blockers, for at least six weeks. At home, the device was placed over the perineum twice a day for 10 minutes using a portable device provided by the manufacturer. After 12 weeks, there was no significant difference in the NIH-CPSI scores between the SEM and placebo groups. On the other hand, subgroup analysis showed that the benefit of SEM was more evident than that of the placebo in patients with a symptom duration of $<12$ months.

Although the precise mechanism of action is not completely understood, acupuncture has been used as a complementary therapeutic option in the management of refractory CP/CPPS in Eastern countries. Several studies have reported that acupuncture may increase the endogenous release of opioids and modulate the sympathetic tone and pain pathways [14,23]. Qin et al. [24] conducted a recent systematic review of acupuncture for the treatment of $\mathrm{CP} / \mathrm{CPPS}$. They reported that it was effective in managing $\mathrm{CP} / \mathrm{CPPS}$.

\section{Tenderness}

The targeting of key modulators in the pain pathway and nerve growth factor (NGF) inhibition has attracted increasing interest. Both modulators have been found to reduce pain in animal models. Tanezumab, a monoclonal antibody against NGF, is an effective agent. Nickel et al. [25] performed a pooled analysis in patients with chronic pelvic pain, including women with interstitial cystitis and men with $\mathrm{CP} / \mathrm{CPPS}$. Pooled analysis showed that tanezumab improved the pain scores. On the other hand, the results were not significant in the male population with CP/CPPS. Therefore, 
tanezumab is considered a promising therapy. Nevertheless, further studies, particularly in the CP/CPPS population, will be needed.

Botulinum neurotoxin-A (BoNT-A) is a toxin familiar to several urologists and is used in different urologic conditions, including neurogenic detrusor overactivity. Falahatkar et al. [26] performed a randomized, double-blind study. They reported statistically significant continuous and stable improvement in the total and subscale scores of the NIH-CPSI during the final six-month evaluation in 60 patients with $\mathrm{CP} / \mathrm{CPPS}$ who received a transurethral intraprostatic injection of BoNT-A or normal saline. Similar results were obtained using other instruments, such as the American Urologic Association (AUA) symptom score, visual analog scale, and quality of life scores. No significant adverse events were recorded except in two patients who developed transient gross hematuria, which was managed conservatively.

\section{EVOLUTION OF PHENOTYPE MAPPING}

With the initial success of including the phenotype domains in the UPOINT system to better classify and treat CP/CPPS, the classification systems for CP/CPPS have been developed. Table 2 lists the diagnostic criteria and possible treatments for the UPOINT phenotypes [15]. Magri et al. [27] evaluated the inclusion of a sexual dysfunction domain to the UPOINT clinical phenotyping system. In this study, 937 European patients with CPPS were classified retrospectively using the modified UPOINTS system ( $\mathrm{S}$, sexual dysfunction domain). The UPOINTS scores correlated with the NIH-CPSI scores in this cohort. Similarly, another study found that the addition of a sexual dysfunction domain improved the quality of life [28]. On the other hand, Samplaski et al. [29] reported that erectile dysfunction did not independently affect the severity of CP/CPPS symptoms or the quality of life.

The UPOINT system focuses on categorizing and individualizing therapy for CP/CPPS via symptom phenotype mapping. The Dimitrakoff, Allsop, Bhai, Brook, Erstad, and Cohen (DABBEC) system is a mechanistically predictive phenotyping system. The two main domains of CP/CPPS in the DABBEC phenotyping system (DPS) are the hypothalamic-pituitary-adrenal domain and the CYP21A2 domain (adrenocortical function). Significant correlations between the specific biomarkers and CP/CPPS have not been elucidated. Unlike the UPOINT system, however, the DPS mechanistic phenotyping system is used to categorize $\mathrm{CP} / \mathrm{CPPS}$ within these domains, which might provide more insight into the pathophysiology of CP/CPPS and new targeted therapies [30].

Cluster analysis is performed to assess the similarities among the apparent heterogeneous phenotypes [29]. This analysis was performed on the UPOINT domains. The results showed two main clusters with homogenous profiles (urinary, organ-specific, and tenderness domains as one cluster, and neurologic, infection, and psychosocial domains as the other). The two homogeneous clusters suggest that the patient groups may differ in terms of their pathophysiology and treatment response [31]. Davis et al. [32] performed a

Table 2. Diagnostic criteria and treatments for UPOINT phenotypes

\begin{tabular}{lll}
\hline \multicolumn{1}{c}{ Domain } & \multicolumn{1}{c}{ Diagnostic criteria } & \multicolumn{1}{c}{ Possible treatment } \\
\hline Urinary & $\begin{array}{l}\text { Bothersome irritative or obstructive urinary symptoms } \\
\text { High postvoid residual }\end{array}$ & $\begin{array}{l}\text { Alpha-blockers } \\
\text { Antimuscarinics }\end{array}$ \\
& & 5-ARI \\
Psychosocial & Clinical depression & Psychologic or psychiatric counseling \\
& Catastrophizing (verbalized helplessness and hopelessness) & Cognitive-behavioral therapy \\
Organ-specific & Prostate tenderness & Quercetin \\
& Symptom relief with voiding & Pollen extract \\
Infection & Positive cultures of prostatic fluid in the absence of UTI & Antibiotics \\
& Urethritis & \\
Neurologic/systemic & Pain outside pelvis & Neuromodulating medications \\
& Neuropathic pain & Acupuncture \\
Tenderness & Pelvic floor dysfunction: spasm & Pelvic physical therapy \\
& Muscle trigger points in pelvis/abdomen & Myofascial release \\
& & Injection therapy \\
\hline
\end{tabular}

UPOINT: Urinary, Psychosocial, Organ-Specific, Infection, Neurological/Systemic, and Tenderness, UTI: urinary tract infection, 5-ARI: 5-alpha reductase inhibitor. 
subtype-based cluster analysis using validated questionnaires and urologic examinations on patients with urologic pelvic pain. The results revealed seven distinct homogeneous clusters (ejaculatory pain/sexual dysfunction, new pain, low pain symptom/distress, prostate disease, testicular pain, long-term genitopelvic pain, and whole-body pain). Interestingly, whole body pain was a separate cluster that is similar to the second cluster based on the UPOINT domains in the Samplaski group (neurologic, infection, and psychosocial). Therefore, there may be a subtype of urologic pelvic pain with a central/systemic pathophysiology.

\section{CONCLUSIONS}

$\mathrm{CP} / \mathrm{CPPS}$ is a condition that can be challenging to treat, and a multimodal approach is commonly required. UPOINT-directed therapy is an approach that simplifies treatment in patients with CPPS. The efficacy of the UPOINT clinical phenotyping system as a diagnostic and treatment tool has been validated in patients with CP/CPPS. The UPOINT system itself may require improvement, and the criteria for each domain have not been fully validated. Nevertheless, this system is considered to be an effective diagnostic and treatment algorithm.

\section{CONFLICT OF INTEREST}

No potential conflict of interest relevant to this article was reported.

\section{REFERENCES}

1. Polackwich AS, Shoskes DA. Chronic prostatitis/chronic pelvic pain syndrome: a review of evaluation and therapy. Prostate Cancer Prostatic Dis 2016;19:132-8.

2. Krieger JN, Nyberg L Jr, Nickel JC. NIH consensus definition and classification of prostatitis. JAMA 1999;282:236-7.

3. Smith CP. Male chronic pelvic pain: an update. Indian J Urol 2016;32:34-9.

4. Shoskes DA, Nickel JC, Rackley RR, Pontari MA. Clinical phenotyping in chronic prostatitis/chronic pelvic pain syndrome and interstitial cystitis: a management strategy for urologic chronic pelvic pain syndromes. Prostate Cancer Prostatic Dis 2009;12:177-83.

5. Magri V, Marras E, Restelli A, Wagenlehner FM, Perletti G. Multimodal therapy for category III chronic prostatitis/chronic pelvic pain syndrome in UPOINTS phenotyped patients. Exp
Ther Med 2015;9:658-66.

6. Hedelin $\mathrm{HH}$. Evaluation of a modification of the UPOINT clinical phenotype system for the chronic pelvic pain syndrome. Scand J Urol Nephrol 2009;43:373-6.

7. Nickel JC, Narayan P, McKay J, Doyle C. Treatment of chronic prostatitis/chronic pelvic pain syndrome with tamsulosin: a randomized double blind trial. J Urol 2004;171:1594-7.

8. Nickel JC, O'Leary MP, Lepor H, Caramelli KE, Thomas H, Hill $\mathrm{LA}$, et al. Silodosin for men with chronic prostatitis/chronic pelvic pain syndrome: results of a phase II multicenter, double-blind, placebo controlled study. J Urol 2011;186: 125-31.

9. Magistro G, Wagenlehner FM, Grabe M, Weidner W, Stief CG, Nickel JC. Contemporary management of chronic prostatitis/ chronic pelvic pain syndrome. Eur Urol 2016;69:286-97.

10. Kong DH, Yun CJ, Park HJ, Park NC. The efficacy of mirodenafil for chronic prostatitis/chronic pelvic pain syndrome in middle-aged males. World J Mens Health 2014;32:145-50.

11. Tripp DA, Nickel JC, Katz L, Krsmanovic A, Ware MA, Santor D. A survey of cannabis (marijuana) use and self-reported benefit in men with chronic prostatitis/chronic pelvic pain syndrome. Can Urol Assoc J 2014;8:E901-5.

12. Gundersen TD, Jørgensen N, Andersson AM, Bang AK, Nordkap L, Skakkebæk NE, et al. Association between use of marijuana and male reproductive hormones and semen quality: a study among 1,215 healthy young men. Am J Epidemiol 2015;182:473-81.

13. du Plessis SS, Agarwal A, Syriac A. Marijuana, phytocannabinoids, the endocannabinoid system, and male fertility. J Assist Reprod Genet 2015;32:1575-88.

14. Sandhu J, Tu HYV. Recent advances in managing chronic prostatitis/chronic pelvic pain syndrome. F1000Res 2017;6: F1000 Faculty Rev-1747.

15. Shoskes DA, Nickel JC. Classification and treatment of men with chronic prostatitis/chronic pelvic pain syndrome using the UPOINT system. World J Urol 2013;31:755-60.

16. Wagenlehner FM, Ballarini $S$, Naber KG. Immunostimulation in chronic prostatitis/chronic pelvic pain syndrome (CP/CPPS): a one-year prospective, double-blind, placebo-controlled study. World J Urol 2014;32:1595-603.

17. Li HS, Wang B, Han L, Wang CH, Xin ZC. [Transperineal ultrasonic therapy for chronic prostatitis]. Zhonghua Nan Ke Xue 2013;19:49-53. Chinese.

18. Zhou Z, Hong L, Shen X, Rao X, Jin X, Lu G, et al. Detection of nanobacteria infection in type III prostatitis. Urology 2008;71: 1091-5.

19. Zhao WP, Zhang ZG, Li XD, Yu D, Rui XF, Li GH, et al. Celecoxib reduces symptoms in men with difficult chronic pelvic pain syndrome (Category IIIA). Braz J Med Biol Res 2009;42:963-7.

20. Bozzini G, Provenzano M, Buffi N, Seveso M, Lughezzani G, Guazzoni G, et al. An observational study of the use of beclomethasone dipropionate suppositories in the treatment 
of lower urinary tract inflammation in men. BMC Urol 2016;16:25.

21. Kotb AF, Ismail AM, Sharafeldeen M, Elsayed EY. Chronic prostatitis/chronic pelvic pain syndrome: the role of an antifungal regimen. Cent European J Urol 2013;66:196-9.

22. Kessler TM, Mordasini L, Weisstanner C, Juni P, da Costa BR, Wiest $R$, et al. Sono-electro-magnetic therapy for treating chronic pelvic pain syndrome in men: a randomized, placebocontrolled, double-blind trial. PLoS One 2014;9:e113368.

23. Blanchet KD. Acupuncture: gaining acceptance in urology: proves effective for a number of chronic conditions. BJU Int 2012;109:ii-iv.

24. Qin Z, Wu J, Zhou J, Liu Z. Systematic review of acupuncture for chronic prostatitis/chronic pelvic pain syndrome. Medicine (Baltimore) 2016;95:e3095.

25. Nickel JC, Mills IW, Crook TJ, Jorga A, Smith MD, Atkinson G, et al. Tanezumab reduces pain in women with interstitial cystitis/bladder pain syndrome and patients with nonurological associated somatic syndromes. J Urol 2016;195(4 Pt 1):942-8.

26. Falahatkar S, Shahab E, Gholamjani Moghaddam K, Kazemnezhad E. Transurethral intraprostatic injection of botulinum neurotoxin type $\mathrm{A}$ for the treatment of chronic prostatitis/chronic pelvic pain syndrome: results of a prospective pilot double-blind and randomized placebo-controlled study. BJU Int 2015;116:641-9.

27. Magri V, Wagenlehner F, Perletti G, Schneider S, Marras E, Naber KG, et al. Use of the UPOINT chronic prostatitis/chronic pelvic pain syndrome classification in European patient cohorts: sexual function domain improves correlations. J Urol 2010;184:2339-45.

28. Davis SN, Binik YM, Amsel R, Carrier S. Is a sexual dysfunction domain important for quality of life in men with urological chronic pelvic pain syndrome? Signs "UPOINT" to yes. J Urol 2013;189:146-51.

29. Samplaski MK, Li J, Shoskes DA. Inclusion of erectile domain to UPOINT phenotype does not improve correlation with symptom severity in men with chronic prostatitis/chronic pelvic pain syndrome. Urology 2011;78:653-8.

30. Allsop SA, Erstad DJ, Brook K, Bhai SF, Cohen JM, Dimitrakoff JD. The DABBEC phenotyping system: towards a mechanistic understanding of CP/CPPS. Nat Rev Urol 2011;8:107-13.

31. Kartha GK, Kerr H, Shoskes DA. Clinical phenotyping of urologic pain patients. Curr Opin Urol 2013;23:560-4.

32. Davis SN, Binik YM, Amsel R, Carrier S. A subtype based analysis of urological chronic pelvic pain syndrome in men. J Urol 2013;190:118-23. 